\title{
Topical Solasodine Rhamnosyl Glycosides Derived from the Eggplant Treats Large Skin Cancers: Two Case Reports
}

\section{Bill E. Cham}

Australasian Medical Research, Devil’s Point Road, Port Vila, Republic of Vanuatu.

Email: bill.cham@gmail.com

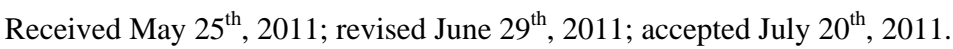

\begin{abstract}
Solasodine rhamnosyl glycosides (BEC) are a new class of antineoplastics that show superior efficacy than many established anticancer drugs as shown by intravenous, intraperitoneal and intralesion administrations. Previous studies have described the efficacy of BEC on nonmelanoma skin cancers by topical application. Two cases are now reported which show that BEC in a cream formulation Curaderm is very effective for the treatment of large nonmelanoma skin cancers that are considered difficult to treat by existing modalities. Moreover, the cosmetic outcomes are very impressive.
\end{abstract}

Keywords: Nonmelanoma Skin Cancers, Solasodine Rhamnosyl Glycosides, Curaderm, Solamargine, Solasonine

\section{Introduction}

There is an alarming increase in skin cancer incidence. In the US alone, more than two million people develop over 3.5 million nonmelanoma skin cancers every year. This constitutes a more than 300 percent increase in cancer incidence since 1992 [1].

Nonmelanoma skin cancers, such as basal cell carcinoma (BCC) and squamous cell carcinoma (SCC), are the most common forms of skin cancer. Though BCCs are rarely life-threatening, they can be disfiguring when not diagnosed and treated in a timely manner. SCCs can metastasize (spread) to distant tissues or organs and are potentially terminal.

The incidence of skin cancer is higher than all other cancers combined and is considered by some as an epidemic.

A variety of treatments are available for nonmelanoma skin cancers with good outcomes, especially if the cancers are detected and treated in the early stages of development. However, there are some serious disadvantages with the most common treatments. Some disadvantages of current treatments are:

- margin around cancer may not be free of cancer

- moderately painful

- slow healing

- scarring

- specialized training by health professionals with ap- propriate facilities

- expensive

- activity restriction after surgery if skin graft or flap is needed

- limited cosmetic results

In addition high recurrence rates of treated skin cancers have been reported [2].

The treatment and management of nonmelanoma skin cancers cost the USA health care system more than US\$1.4 billion per year and this value is increasing dramatically each year.

There is a need for novel treatments for nonmelanoma skin cancers. It has previously been shown in a large number of studies that the glycoalkaloids solasodine rhamnosyl glycosides (SRGs) induce apoptosis in a wide variety of cancer cells [3-7]. SRGs are present in a diversity of solanaceous plants such as the Devil's Apple (Solanum linnaeanum) and Eggplant (S. melongena). SRGs display specificity towards cancer cells when compared with normal cells and the unique mode of action has been described [8,9]. Anticancer therapies with SRGs in animals and humans have been used intravenously [10], intraperitoneally [11], intralesionally [12] and topically [13-17]. A constant mixture of SRGs, known as BEC, consisting of solasodine containing triglycosides solasonine ( $\beta$-solatriose) (33\%), solamargine $(\beta$-chacotriose) (33\%), and di-and monoglycosides (34\%), are present in 
a cream formulation which contains $0.005 \%$ BEC (Curaderm). Curaderm is reportedly effective for treating nonmelanoma skin cancers as shown by uncontrolled [13-16] and controlled studies [17]. Here, two cases of large skin cancers treated with the cream formulation Curaderm are reported.

\section{Case Reports}

\subsection{Case Report 1}

A 68 year old farmer was referred for consultation because he had a large basal cell carcinoma (BCC). Dermatologists and surgeons had recommended surgical excision and radiotherapy followed by surgical reconstruction with skin grafting. The patient who had this
BCC for at least 3 years elected to treat the lesion with the cream formulation Curaderm. The patient exhibited a large lesion, $4 \mathrm{~cm} \times 4 \mathrm{~cm} \times 2 \mathrm{~cm}$, on the right side of his face next to his ear (Figure 1 top row). Histological analysis of a biopsy determined that it was a BCC.

\subsection{Case Report 2}

A 63 year old retired man had a histologically confirmed squamous cell carcinoma (SCC), $4 \mathrm{~cm}$ in diameter, on his head (Figure 2(a)). This patient who had this SCC for at least 2 years refused other treatments and decided to have Curaderm therapy.

\subsection{Materials and Methods}

The cream formulation Curaderm is available to patients
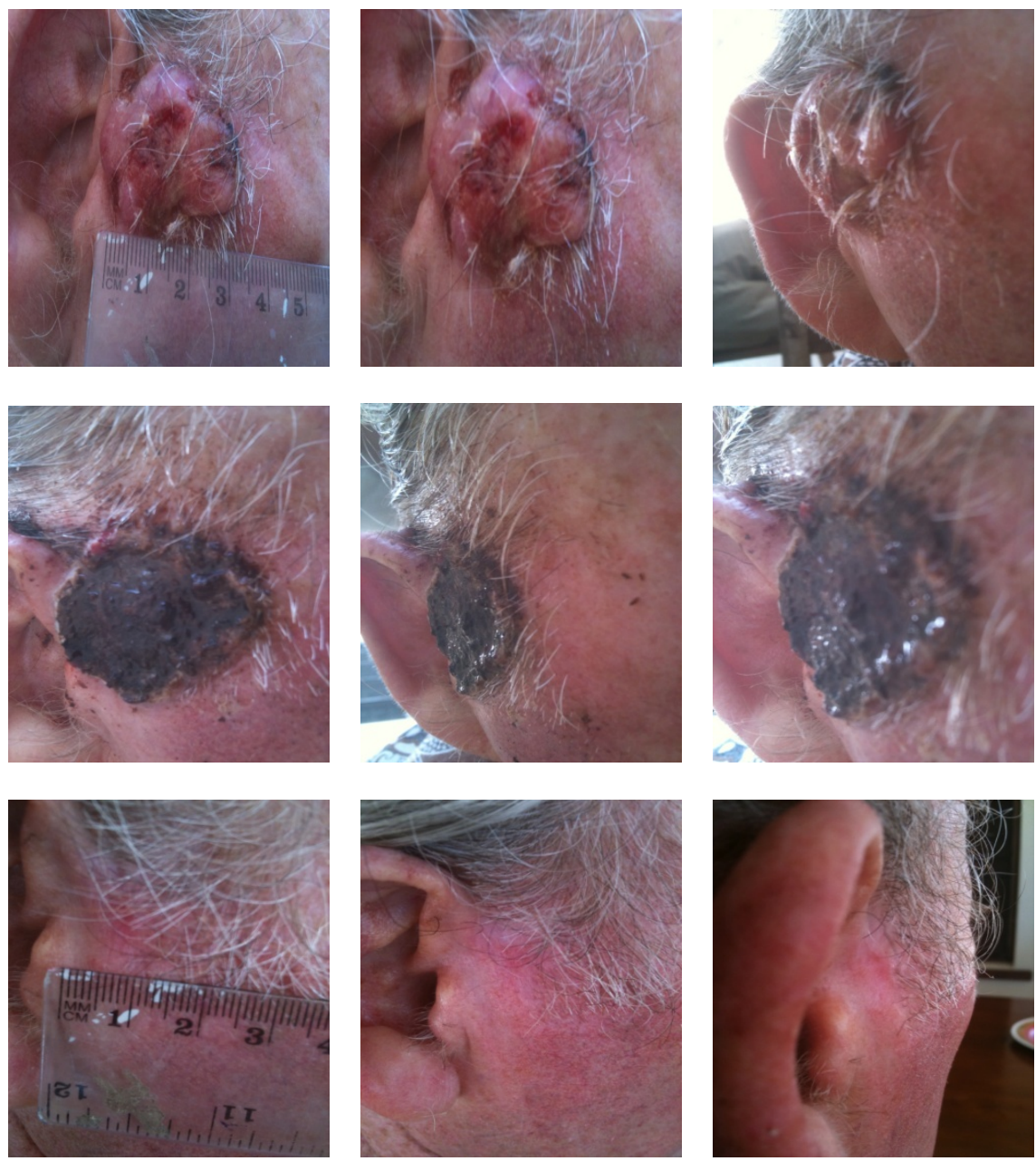

Figure 1. An extensive protruding $(4 \mathrm{~cm} \times 4 \mathrm{~cm} \times 2 \mathrm{~cm})$ BCC with central ulceration and raised curly borders on the right side of his face next to his ear is seen in patient 1 (top row). Treatment with Curaderm resulted in rapid break-down of the tumour and after 2 weeks of treatment the lesion was reduced to about a half of the original size. Minor bleeding had occurred during this treatment period (middle row). After 14 weeks of treatment the lesion was clinically eliminated. Normal skin cells had replaced the tumour and the cosmetic end result was excellent, with no scar tissue formation. Even the hairs had regrown where the tumour was originally (bottom row). 
in several countries. Curaderm contains the glycoalkaloids BEC at $0.005 \%$ as a topical cream formulation. The cream was applied twice daily (when possible every 12 hrs) under occlusive dressing (micropore paper tape) until the lesion had clinically regressed.

\section{Results}

Figure 1 top row shows the extent of the BCC just before treatment with Curaderm. The lesion responded rapidly to the treatment. The middle row of Figure 1 shows the appearance of the lesion after 2 weeks of treatment. It can be seen that minor bleeding had occurred during treatment. The bottom row of Figure 1 shows the treated area after 14 weeks of Curaderm therapy. The lesion was eliminated by the treatment and the cosmetic end result was outstanding. Note, there was no scar tissue and the hairs had regrown where the lesion was originally. This patient did not experience side effects during Curaderm therapy other than some bleeding had occurred. There has been no recurrence one year after treatment.

Figure 2(a) shows a large SCC before treatment. This lesion sometimes oozed exudates. Figure 2(b) shows the lesion after 3 weeks of Curaderm treatment, the lesion appeared larger at this stage. Another 3 weeks of treatment shows that the lesion was much "cleaner" and was starting to fill in with normal tissue (Figure 2(c)). Figure 2(d) shows that after another 3 weeks and that during treatment more normal skin tissue was replacing the cancer tissue. After another 2 weeks of treatment the lesion was almost completely healed (Figure 2(e)). After a total of 14 weeks of treatment, the lesion had regressed and normal skin tissue had replaced the lesion. There was no scar tissue at the completion of the treatment (Figure 2(f)). This patient experienced mild itching and stinging surrounding the treated lesion for the first week of Curaderm therapy. There has been no recurrence five years after treatment.

\section{Discussion}

SRGs have previously been shown to have good anticancer properties and are superior to other anticancer agents such as taxol, cisplatin, gemcitabine, camptothecin, vinblastine, methotrexate, 5-fluorouracil, epirubicin and cyclophosphamide $[12,18,19]$. The mode of action of SRGs is unlike any current antineoplastic agent. Specific receptors for the SRGs present only on cancer cells but not normal cells are the first step of events that lead to apoptosis in cancer cells only, and this may explain why during treatment the cancer cells were being eliminated and normal cells were replacing the killed cancer cells

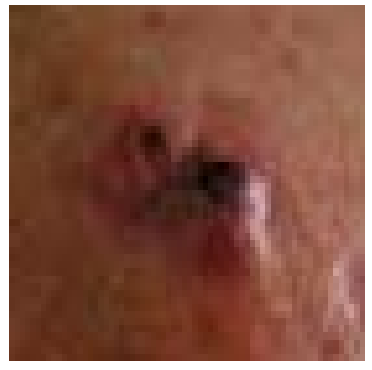

(a)

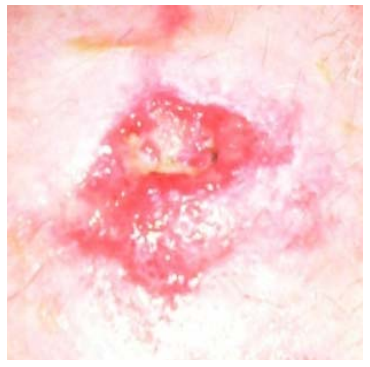

(d)

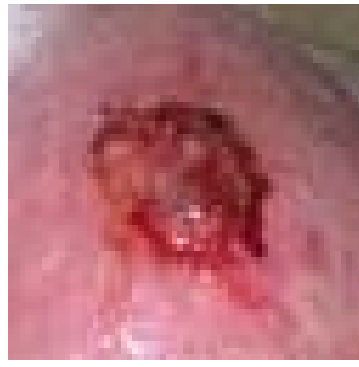

(b)

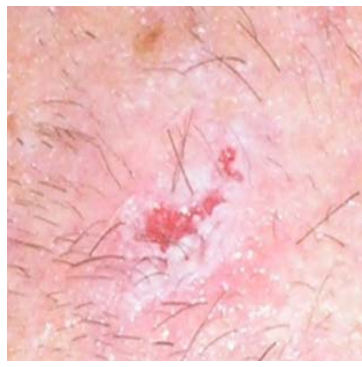

(e)

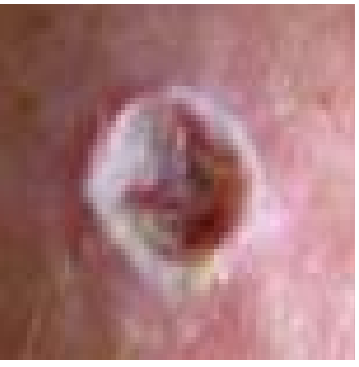

(c)

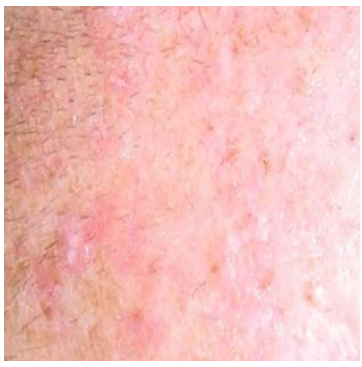

(f)

Figure 2. A large (4 cm diameter) sometimes oozing SCC on the head of patient 2 (a). Three weeks after Curaderm treatment the lesion appeared to have increased in size (b). Another 3 weeks treatment resulted in the lesion appearing much "cleaner" with some replacement of cancer tissue with normal tissue (c). After another 3 weeks treatment the lesion was much smaller and normal skin tissue had replaced the treated cancerous tissue whilst continually being treated with Curaderm (d). Two weeks later, the treated lesion was in the final stages of healing (e). After a total of 14 weeks treatment the entire lesion was clinically removed and there was no scar tissue at the completion of treatment (f). 
with no scar tissue being formed.

The two cases presented here are large and anatomically difficult to treat lesions. There is little doubt that the cosmetic end result of this type of treatment is at least, or more likely, superior to other available treatments. At the completion of treatment, it could not be distinguished where the tumours once were! Other published studies have shown that recurrences are low with extended follow-up periods [13-17], and no major side effects during Curaderm therapy other than mild itching and transient burning surrounding the treated lesion had occurred. In addition it has been reported that Curaderm therapy had no adverse effects on the liver, kidneys or haematopoietic system $[17,18]$.

Phase I clinical trials with BEC at various concentrations up to $50 \%$ in cream formulations were shown to be very safe. There were no changes observed in vital signs. Haematological, biochemical and urinanalytical parameters did not alter by topical application of BEC in such cream formulations $[13,16]$. It was subsequently shown that very low concentrations of BEC, as low as $0.005 \%$ BEC, were effective in treating skin cancers [13-17]. However, in order to obtain efficacy with this very low concentration of BEC in a cream formulation Curaderm ${ }^{\text {BEC5 }}$, salicylic acid (10\%) and urea (5\%) had to be added to the cream. These substances acted as keratolytic agents which enabled BEC to have access to the cancer cells. The stinging and slight burning effects sometimes experienced whilst using Curaderm ${ }^{\mathrm{BEC} 5}$ therapy, are caused by these keratolytic agents. Double-blind, randomized, placebo-controlled, parallel group, multicentre studies established that no significant patterns of change for the full blood count, biochemistry and urinanalysis parameters were observed when the BEC groups at $0.005 \%$ were compared with the placebo control groups. The adverse effects were similar in both the BEC groups and the placebo control groups suggesting that salicylic acid and urea and not BEC were causing the reported adverse effects. It was concluded that BEC exerted antineoplastic activity and the excipients salicylic acid and urea were responsible for the sometimes transient adverse effects of stinging or slight burning [17].

Topical treatment with Curaderm should be considered as an additional therapy for the treatment of nonmelanoma skin cancers. Curaderm treatment improved the quality of life for these patients.

\section{Conclusions}

The incidence of skin cancer is higher than all other forms of cancers combined and skin cancer rates are increasing in epidemic proportions.

New treatments are currently being explored to add to the existing treatments of this disease.

Here it is shown that topical application of a cream Curaderm, containing SRGs in the form of a constant mixture, BEC, is amazingly effective for treating large nonmelanoma skin cancers with incredible cosmetic results.

\section{REFERENCES}

[1] H. W. Rogers, N. A. Weinstock, A. R. Harris, M. R. Hinckley, S. R. Feldman, A. B. Fleischer and B. M. Coldiron, "Incidence Estimate of Nonmelanoma Skin Cancer in the United States," Archives of Dermatology, Vol. 146, No. 3, 2010, pp. 283-287.

doi:10.1001/archdermatol.2010.19

[2] L. A. E. Sussman and D. F. Liggins, "Incompletely Excised Basal Cell Carcinoma a Management Dilemma?” Australian and New Zealand Journal of Surgery, Vol. 66, No. 5, 1996, pp. 276-278. doi:10.1111/j.1445-2197.1996.tb01184.X

[3] K. W. Kuo, S. H. Hsu, Y. P. Li, W. L. Lin, L. F. Liu, L. C. Chang, C. C. Lin, C. N. Lin and H. M. Sheu, “Anticancer Activity Evaluation of the Solanum Glycoalkaloid Solamargine. Triggering Apoptosis in Human Hepatoma Cells," Biochemical Pharmacology, Vol. 60, No. 12, 2000, pp. 1865-1873. doi:10.1016/S0006-2952(00)00506-2

[4] C. H. Liang, L. F. Liu, L. Y. Shiu, Y. S. Huang, L. C. Chang and K. W. Kuo, "Action of Solamargine on TNFs and Cisplatin-Resistant Human Lung Cancer Cells,” Biochemical and Biophysical Research Communications, Vol. 322, No. 3, 2004, pp. 751-758.

[5] L. Y. Shiu, L. C. Chang, C. H. Liang, Y. S. Huang, H. M. Sheu and K. W. Kuo, "Solamargine Induces Apoptosis and Sensitizes Breast Cancer Cells to Cisplatin," Food and Chemical Toxicology, Vol. 45, No. 11, 2007, pp. 2155-2164. doi:10.1016/j.fct.2007.05.009

[6] L. Y. Shiu, C. H. Liang, Y. S. Huang, H. M. Sheu and K. W. Kuo, "Downregulation of HER2/neu Receptor by Solamargine Enhances Anticancer Drug-Mediated Cytotoxicity in Breast Cancer Cells with High-Expressing HER2/neu," Cell Biology and Toxicology, Vol. 24, No. 1, 2008, pp. 1-10. doi:10.1007/s10565-007-9010-5

[7] L. Sun, Y. Zhao, H. Yuan, X. Li, A. Cheng and H. Lou, "Solamargine, a Steroidal Alkaloid Glycoside, Induces Oncosis in Human K562 Leukemia and Squamous Cell Carcinoma KB Cells,” Cancer Chemotherapy and Pharmacology, Vol. 65, No. 4, 2010, pp. 1125-1130.

[8] B. E. Cham and B. Daunter, "Solasodine Glycosides. Selective Cytotoxicity for Cancer Cells and Inhibition of Cytotoxicity by Rhamnose in Mice with Sarcoma 180," Cancer Letters, Vol. 55, No. 3, 1990, pp. 221-225. doi:10.1016/0304-3835(90)90122-E

[9] B. Daunter and B. E. Cham, "Solasodine Glycosides. In Vitro Preferential Cytotoxicity for Human Cancer Cells," Cancer Letters, Vol. 55, No. 3, 1990, pp. 209-220. 


\section{doi:10.1016/0304-3835(90)90121-D}

[10] M. Millward, A. Powell, P. Daly, S. Tyson, R. Ferguson and S. Carter, "Results of Phase I Clinical Trials of Coramsine in Patients with Advanced Solid Tumours," Journal of Clinical Oncology, Vol. 24, No. 18S, 2006, p. 2070.

[11] B. E. Cham, M. Gilliver and L. Wilson, "Antitumour Effects of Glycoalkaloids Isolated from Solanum Sodomaeum L.,” Planta Medica, Vol. 53, No. 1, 1987, pp. 3436. doi:10.1055/s-2006-962612

[12] B. E. Cham, "Cancer Intralesion Chemotherapy with Solasodine Rhamnosyl Glycosides,” Research Journal of Biological Sciences, Vol. 3, No. 9, 2008, pp. 1008-1017.

[13] B. E. Cham, "Solasodine Rhamnosyl Glycosides Specifically Bind Cancer Cell Receptors and Induce Apoptosis and Necrosis. Treatment for Skin Cancer and Hope for Internal Cancers," Research Journal of Biological Sciences, Vol. 2, No. 4, 2007, pp. 503-514.

[14] B. E. Cham, B. Daunter and R. Evans, "Topical Treatment of Malignant and Premalignant Skin Cancers by Very Low Concentrations of a Standard Mixture of Solasodine Glycosides," Cancer Letters, Vol. 59, No. 3, 1991, pp. 183-192. doi:10.1016/0304-3835(91)90140-D
[15] B. E. Cham, "Solasodine Rhamnosyl Glycosides in a Cream Formulation Is Effective for Treating Large and Troublesome Skin Cancers," Research Journal of Biological Sciences, Vol. 2, No. 7, 2007, pp. 749-761.

[16] B. E. Cham and M. M. Meares, "Glycoalkaloids from Solanum sodomaeum L. Are Effective in the Treatment of Skin Cancers in Man," Cancer Letters, Vol. 36, No. 2, 1987, pp. 111-118. doi:10.1016/0304-3835(87)90081-4

[17] S. Punjabi, L. J. Cook, P. Kersey, R. Marks and R. Cerio, "Solasodine Glycoalkaloids: A Novel Topical Therapy for Basal Cell Carcinoma. A Double-Blind, Randomized, Placebo-Controlled, Parallel Group, Multicentre Study," International Journal of Dermatology, Vol. 47, No. 1, 2008, pp. 78-82. doi:10.1111/j.1365-4632.2007.03363.x

[18] K. W. Kuo and C. N. Lin, "Pharmacological Composition for Treating Cancer Cells,” United states Patent, No. US 9/435521, 1999.

[19] L. Y. Shiu, L. C. Chang, C. H. Liang, Y. S. Huang, H. M. Sheu and K. W. Kuo, "Solamargine Induces Apoptosis and Sensitizes Breast Cancer Cells to Cisplatin,” Food and Chemical Toxicology, Vol. 45, No. 11, 2007, pp. 2155-2164. doi:10.1016/j.fct.2007.05.009 